\title{
RHEOLOGICAL SLS MODEL. DYNAMIC PARAMETERS OF THE MECHANICAL SYSTEMS WITH VISCOUS DAMPING. PART 2: TRANSMISSIBILITY FACTOR AND ISOLATION DEGREE
}

\author{
Assoc. Prof. Dr. Eng. Nicusor DRAGAN \\ MECMET - The Research Center of Machines, \\ Mechanic and Technological Equipments \\ Engineering and Agronomy Faculty of \\ Braila \\ "Dunarea de Jos" University of Galati
}

\begin{abstract}
The paper presents the dynamic model of a 1DOF elastic mechanical system with viscous damping rheologically modeled as SLS (Standard Linear Solid) model. The SLS rheological model is a Maxell model in parallel with a Hooke model. The perturbation force $F_{0}$ sin $\omega t$ is transmitted to the base through both of the elements (Hooke and Maxwell). The analyzed dynamic characteristics are the transmissibility ratio $T(\Omega, \zeta)$ and the isolation degree $I(\Omega, \zeta)$, function of the viscous damping ratio $\zeta$ and the relative frequency $\Omega$.
\end{abstract}

KEYWORDS: 1DOF, viscous damping, SLS model, transmissibility ratio, isolation degree

\section{INTRODUCTION. THE \\ DYNAMIC MODEL OF 1DOF MECHANICAL SYSTEM WITH SLS VISCOUS DAMPING}

Considering the calculus scheme of the 1DOF mechanical system with SLS viscous damping support from figure 1 , perturbed by the variable harmonic force $F(t)=F_{0} s i n t$, the structural linear characteristics are:

the supported mass $m$;

- the visco-elastic characteristics of SLS rheological model:

-the stiffness coefficient $k^{1}$ of the Hooke model

-the stiffness coefficient $k^{2}=N k^{1}$ of the Maxwell model

-the viscous damping coefficient $b^{2}$ of the Maxwell model

The kinematic parameters of the mechanical system are:

- displacement ${ }^{z} f$, speed $\dot{z}_{f}$ and acceleration $\ddot{z}_{f}$ of mass $m$

- displacement $y$ and speed $\dot{y}$ of the connection point of the Maxwell model (between the Hooke model and the Newton model).

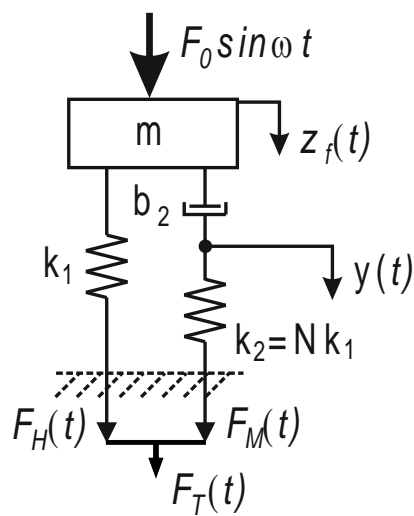


Fig. 1. Model of 1DOF mechanical system with viscous damping (SLS rheological model) perturbed by a harmonic force [1] [2] [3]

\section{TRANSMISSIBILITY RATIO}

The perturbation harmonic force is transmitted from the mass $m$ to the base through the Hooke model and the Maxwell model. The transmitted force to the base is the sum of the Hooke force and the Maxwell force as follows [4] [5]:

$$
F_{T}(t)=F_{H}(t)+F_{M}(t)
$$

Since the components of SLS rheological model are linear, the kinematic and dynamic parameters of the system have harmonic time variation, with the same angular frequency $\omega$ as the perturbation force, with different values for the phase shift [4] [5]:

$$
\begin{aligned}
& z_{f}(t)=A_{f} s\left(\omega t-\varphi_{O}\right) \\
& y(t)=A_{Y} s(\omega t-\alpha) \\
& F_{T}(t)=F_{O T} s(\omega \Delta t-\beta)
\end{aligned}
$$

The moving equations and the transmitted force can be written as follows [1] [2] [3]:

$$
\left\{\begin{array}{l}
m \ddot{z}+b_{2}\left(\dot{z}_{f}-\dot{y}\right)+k_{1} z_{f}=F_{0} s \quad i \omega n \\
b_{2}\left(\dot{z}_{f}-\dot{y}\right)=k_{2} y \\
F_{T}(t)=k_{1} z_{f}+k_{2} y
\end{array}\right.
$$

Taking into consideration (2) (3) (4), the transmitted force to the base is:

$$
\begin{aligned}
& F_{0 T} s \quad(\dot{\omega} t-n \beta)= \\
& =k_{1} A_{f} s \quad\left(\dot{\omega} t-n \varphi_{0}\right)+k_{2} A_{Y} s \quad(\dot{\omega} t-n \alpha)
\end{aligned}
$$

The amplitude $F_{O T}$ of the transmitted force can be written

$$
F_{0 T}=F_{0} \sqrt{\frac{1+\left(\frac{2 \zeta \Omega}{N}\right)^{2}(N+1)^{2}}{\left(1-\Omega^{2}\right)^{2}+\left(\frac{2 \zeta \Omega}{N}\right)^{2}\left(N+1-\Omega^{2}\right)^{2}}}(7)
$$

where: $N$ is the stiffness coefficients ratio $\zeta$ - viscous damping ratio (Maxwell model)

$$
\begin{gathered}
\Omega=\frac{\omega}{p} \text { - relative (angular) frequency } \\
p \text { - natural frequency (Hooke model) }
\end{gathered}
$$

The amplitude of the transmitted force $F_{0 T}$ can be written function of the amplitude of the perturbation force $F_{0 T}$ and the transmissibility ratio $T(\Omega, \zeta, N)$ as follows:

$$
\begin{gathered}
T(\Omega, \zeta, N)=\sqrt{\frac{1+\left(\frac{2 \zeta \Omega}{N}\right)^{2}(N+1)^{2}}{\left(1-\Omega^{2}\right)^{2}+\left(\frac{2 \zeta \Omega}{N}\right)^{2}\left(N+1-\Omega^{2}\right)^{2}}} \\
F_{O T}=F_{O} \cdot T(\Omega, \delta, N)
\end{gathered}
$$

\section{ISOLATION DEGREE}

Function of transmissibility ratio, the isolation degree is:

$$
I=(1-T) \times 1 \quad 0[\mathscr{6}]
$$

\section{TRANSMISSIBILITY RATIO} DIAGRAMS OF THE MECHANICAL SYSTEM WITH SLS MODEL

Figures 2 to 8 show the transmissibility ratio diagrams function of the relative frequency $\Omega$ with parametric variables $\zeta$ and $N$.

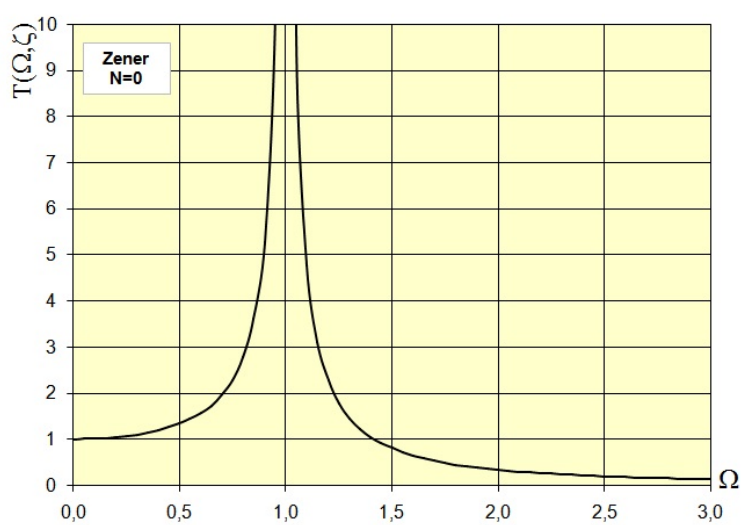

Fig. 2. Transmissibility SLS model $-\mathrm{N}=0$ (Hooke model) 


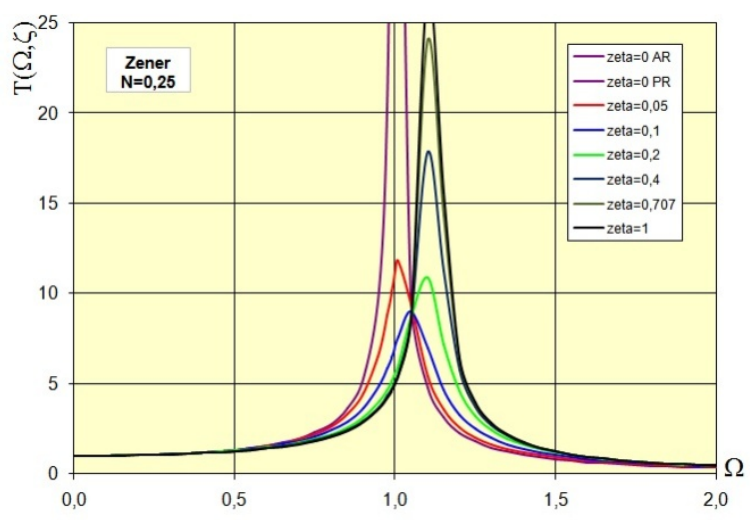

Fig. 3. Transmissibility SLS model $-\mathrm{N}=0.25$

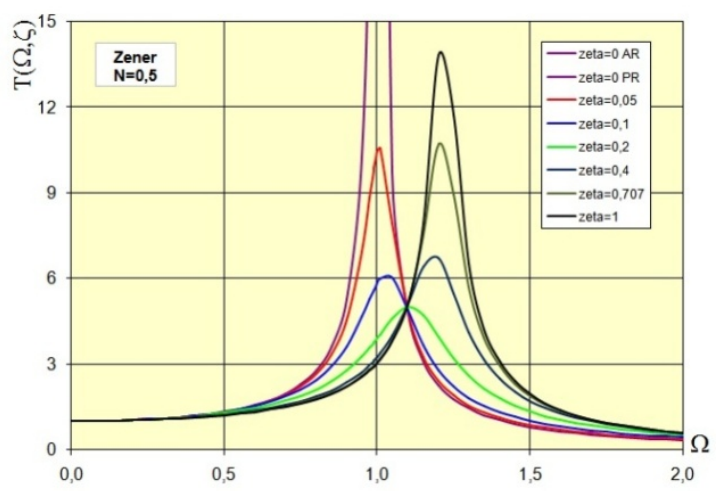

Fig. 4. Transmissibility SLS model - N=0.5

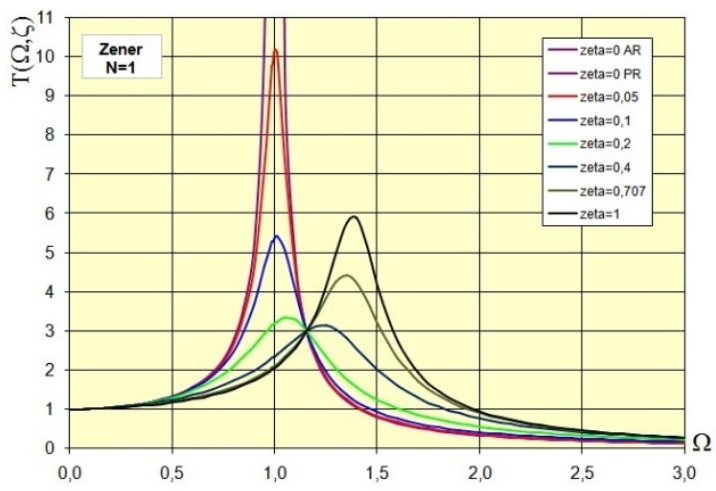

Fig. 5. Transmissibility SLS model - N=1

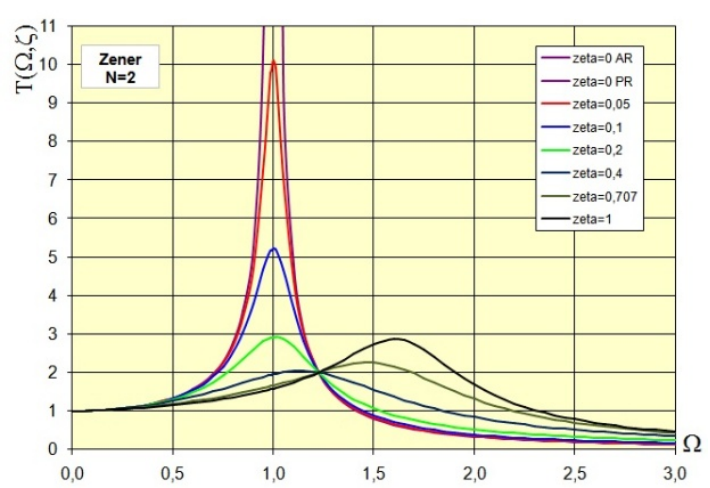

Fig. 6. Transmissibility SLS model - $\mathrm{N}=2$

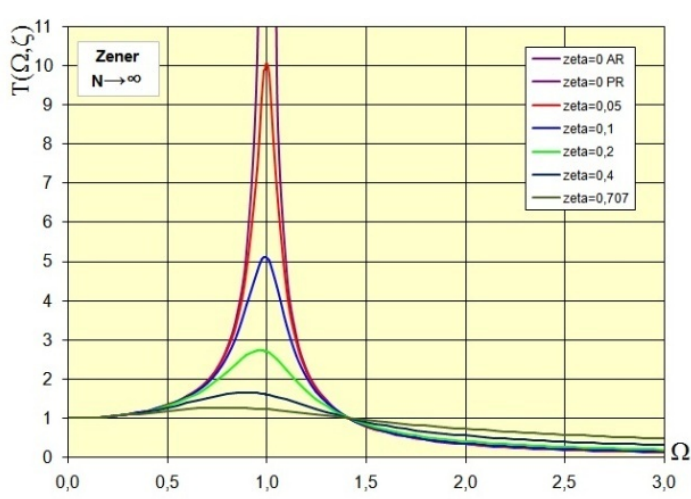

Fig. 7. Transmissibility SLS model - N $\rightarrow \infty$ (Voigt-Kelvin model)

\section{ISOLATION DEGREE}

DIAGRAMS OF THE MECHANICAL SYSTEM WITH SLS MODEL

Figures 8 to 13 show the isolation degree diagrams function of the relative frequency $\Omega$ for different values of the parametric variables $\zeta$ and $N$.

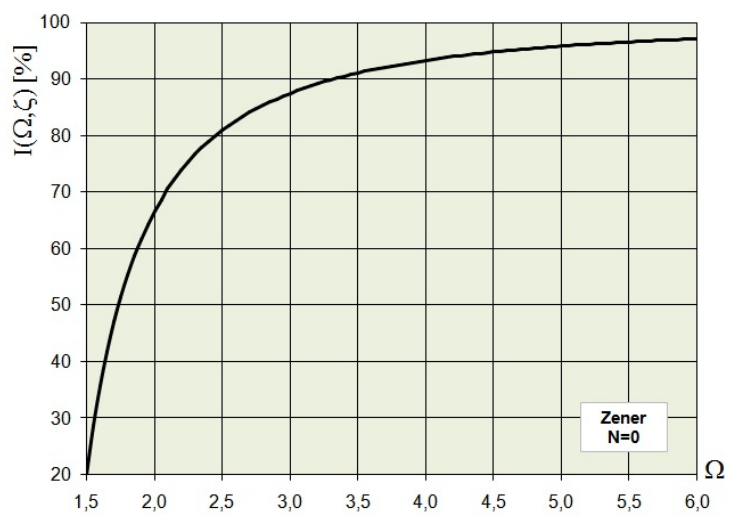

Fig. 8. Isolation degree SLS model - $N=0$ (Hooke model)

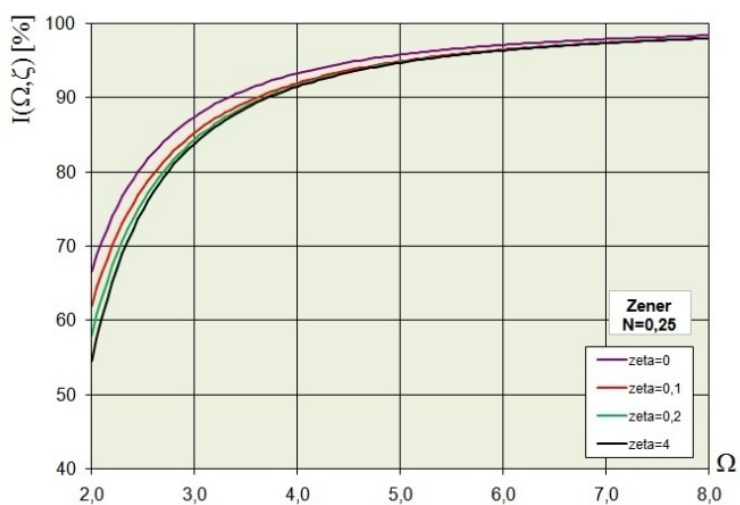

Fig. 9. Isolation degree SLS model - N=0.25 


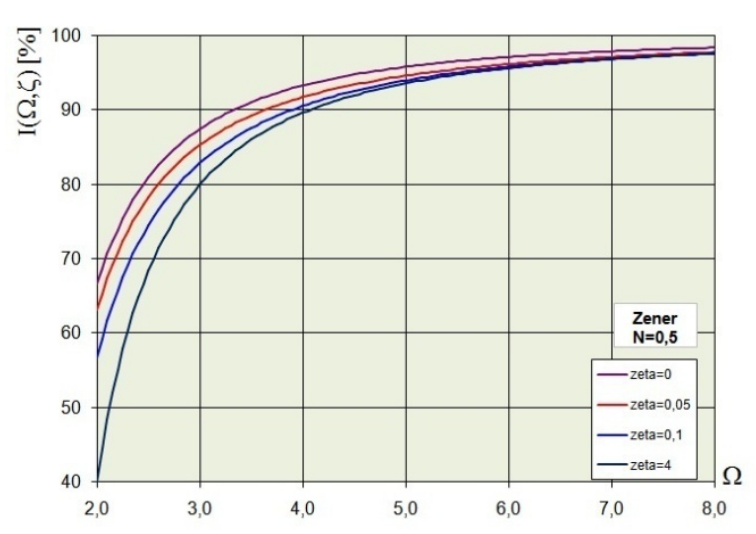

Fig. 10. Isolation degree SLS model $-\mathrm{N}=0.5$

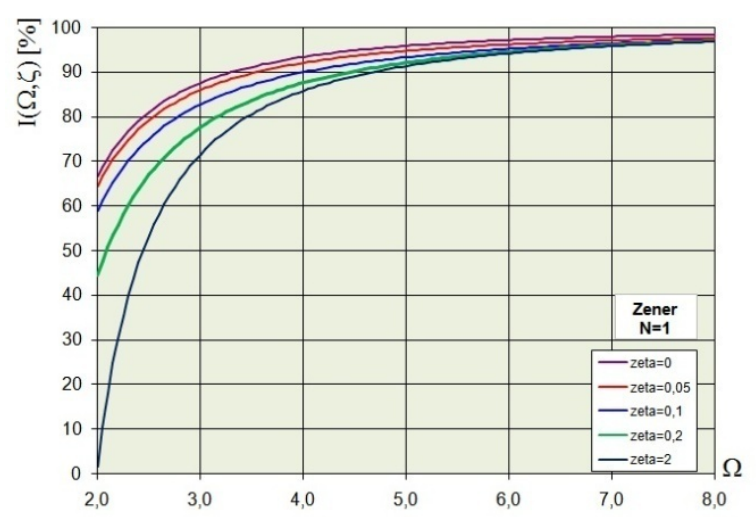

Fig. 11. Isolation degree SLS model - N=1

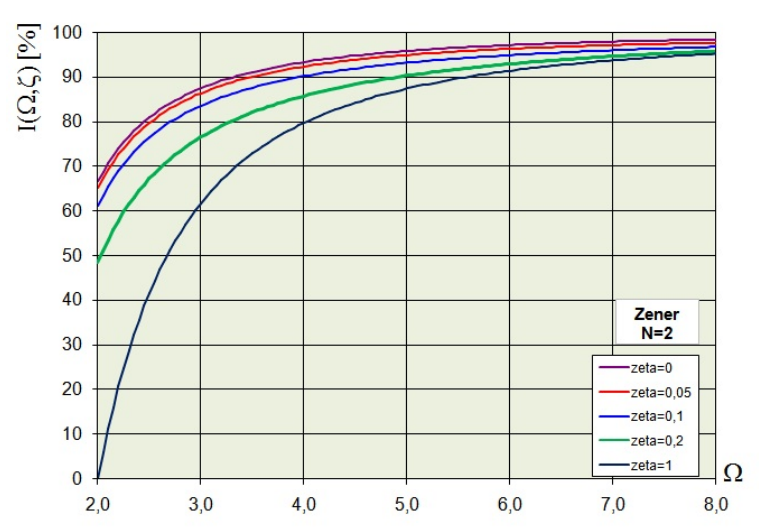

Fig. 12. Isolation degree SLS model - N=2

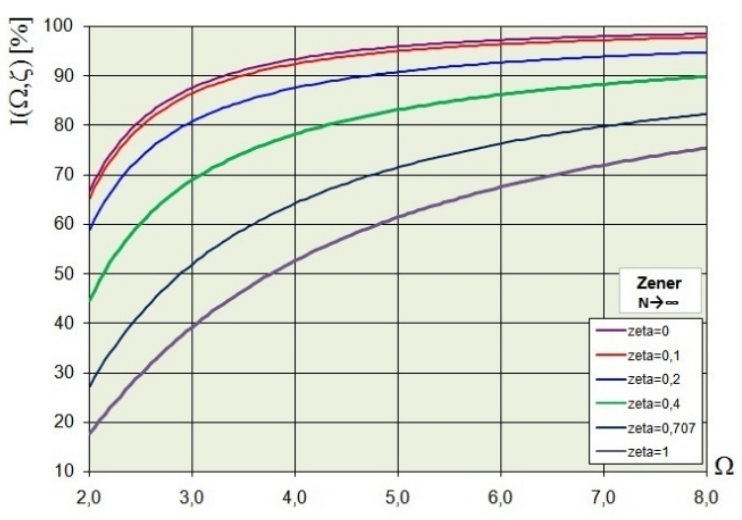

Fig. 13. Isolation degree SLS model - $\mathrm{N} \rightarrow \infty$ (Voigt-Kelvin model)

\section{CONCLUSIONS}

if $N=0 / k_{2}=0$ or $\zeta=0$, SLS model becomes the Hooke model; the transmissibility ratio diagram is shown in figure 2; the transmissibility formula becomes:

$$
T_{N=0}(\Omega) \equiv T_{\zeta=0}(\Omega)=\frac{1}{\left|1-\Omega^{2}\right|}
$$

if $N \rightarrow \infty / k_{2}>>k_{1}$ (the spring from the Maxwell model becomes a rigid connection), SLS rheological model becomes the VoigtKelvin model; the transmissibility ratio diagram is shown in figure 7; the transmissibility ratio formula becomes:

$$
T_{N \rightarrow \infty}(\Omega, \zeta)=\sqrt{\frac{1+4 \zeta^{2} \Omega^{2}}{\left(1-\Omega^{2}\right)^{2}+4 \zeta^{2} \Omega^{2}}}
$$

for the Hooke rheological model, the maximum value for transmissibility ratio is obtained for $\Omega=1$, see figure 2 ;

- for the complex SLS rheological model, the maximum values for transmissibility ratio depends on the stiffness coefficients ratio $N$ (figures 3 to 6); the maximum values are obtained for $\Omega>1$;

the isolation degree depends on the parametric variables $\zeta$ and $N$;

$\checkmark$ we can obtain good isolation degrees (I>95\%) only for bigger relative frequency $(\Omega>3,5 \ldots 4,0)$ and only if viscous damping is smaller than 0.2 .

\section{REFERENCES}

[1] Dragan, N., Dynamic analysis of the parameters of the mechanical systems with structural damping. Viscoelastic SLS model. Part 1: Amplitude factor, The Annals of "Dunarea de Jos" University of Galati, 
Fascicle XIV Mechanical Engineering, ISSN 12245615, Galati, 2016

[2] Dragan, N., Dynamic analysis of the parameters of the mechanical systems with structural damping. Viscoelastic SLS model. Part 2: Transmissibility factor and isolation degree, The Annals of "Dunarea de Jos” University of Galati, Fascicle XIV Mechanical Engineering, ISSN 1224-5615, Galati, 2016

[3] Spanu (Stefan), G.C., Dragan, N., Analysis of the transmissibility ratio and the isolation degree of the vibration for mechanical systems with structural damping. Zener model, Synthesis of Theoretical and Applied Mechanics, ISSN 2068-6331, Bucharest, 2017

[4] Bratu, P., Elastic Systems Vibrations, Technical Publishing House, Bucharest, 2000.

[5] Bratu, P., Bearing Elastic Systems for Machines and Equipment, Technical Publishing House, Bucharest, 1990 